\title{
Combinatorial bounds on connectivity for dominating sets in maximal outerplanar graphs
}

\author{
Santiago Canales ${ }^{2}$ \\ Department of Applied Mathematics \\ Comillas Pontificial University, ICAI, Madrid, Spain \\ Irene Castro ${ }^{3}$ \\ DMATIC, Technical University of Madrid, Madrid, Spain \\ Gregorio Hernández ${ }^{1,4}$ \\ DMATIC, Technical University of Madrid, Madrid, Spain \\ Mafalda Martins ${ }^{1,5}$ \\ CIDMA - Department of Mathematics \\ University of Aveiro, Aveiro, Portugal
}

\begin{abstract}
In this article we study some variants of the domination concept attending to the connectivity of the subgraph generated by the dominant set. This study is restricted to maximal outerplanar graphs. We establish tight combinatorial bounds for connected domination, semitotal domination, independent domination and weakly connected domination for any $n$-vertex maximal outerplaner graph.
\end{abstract}




\section{Introduction}

Given a graph $G=(V, E)$ a dominating set is a set $S \subseteq V$ such that every vertex not in $D$ is adjacent to a vertex in $D$. The domination number $\gamma(G)$ is the number of vertices in a smallest dominating set for $G$. In graph theory, dominanting set problems have received much attention in numerous articles and books, being the fundamental reference the book of Haynes, Hedetniemi and Slater [7], where some variants of domination are analyzed that take into account the connectivity of the subgraph generated by the dominant set. In recent years it has received special attention the problem of domination in outerplanar graphs (e.g., $[1,2,11]) \mathrm{A}$ graph is outerplanar if it has a crossingfree embedding in the plane such that all vertices are on the boundary of its outer face (the unbounded face). An outerplanar graph is maximal if it is not possible to add an edge such that the resulting graph is still outerplanar. A maximal outerplanar graph embedded in the plane corresponds to a triangulation of a polygon. The works mentioned above continued the work started by Matheson and Tarjan [9], where the authors proved that the domination number of a triangulated disc of order $n \geq 3$ is at most $\frac{n}{3}$. A triangulated disc, or triangulation graph, is a plane graph such that all its faces, except the infinite face, are triangles. In this article we establish tight combinatorial bounds for the following domination variants in maximal outerplanar graphs: connected, semitotal, weakly connected and independent. All these variants (and total domination) refers to connectivity of dominating sets. Weakly connected domination was introduced by Grossman [6] and semitotal domination by Goddard et al. [5]. The total domination variant has recently been studied, from a combinatorial point of view by Dorfling et al. [3]. They show that a maximal outerplanar graph of order $n \geq 5$ has total domination number at most $\frac{2 n}{5}$, apart from two exceptions.

In the next section we describe the terminology that will be used throughout this paper and the relation between the different parameters of connectivity and domination in a maximal outerplanar graph. In sections 3 and 4 we present the obtained results for connected and independent dominations and

1 The third author was partially supported by Project MINECO MTM2015-63791-R. The fourth author was supported in part by the Fundação para a Ciência e Tecnologia, through CIDMA - Center for Research and Development in Mathematics and Applications, within project UID/MAT/04106/2013 and by the FCT grant SFRH/BPD/66431/2009.

2 Email: scanales@icai.comillas.edu

3 Email: irene.castro.delgado@alumnos.upm.es

4 Email: gregorio@fi.upm.es

5 Email: mafalda.martins@ua.pt 
for weakly connected and semitotal dominations, respectively. In section 5 we describe some algorithmic results.

\section{Parameters of Connectivity and Domination}

Let $G$ be a graph of order $n$ and $S$ a dominating set of $G$. By imposing different connectivity conditions to the subgraph generated by $S, G[S]$, we have some variants on domination. In this article we will study the following ones: connected domination, if $G[S]$ is connected; independent domination, if $G[S]$ is formed by isolated vertices; weakly connected domination, if the edges not incident to any vertex of $S$ do not separate $G$ and semitotal domination, if for every $u \in S$ there is a $v \in S$ such that $\operatorname{dist}(u, v) \leq 2$. Now we are going to define the notation that we use in a general way. Let $H$ designate any property on the connectivity of $G[S]$, the subgraph generated by the dominant set $S$. The minimum number of elements in a dominant set verifying the property $H$ in a graph $G$ is called domination number under $H$ of $G$ and it is denoted by $h(G)$. That is,

$$
h(G)=\min \{|S|: S \text { is a domination set of } G \text { that satifies } H\} .
$$

In the combinatorial study of any of the variants of domination, which we indicated generally by the $H$ condition, our goal is to determine bounds for

$$
h(n)=\max \{h(G): G \text { is a maximal outerplanar graph with } n \text { vertices }\} .
$$

In the following sections we are going to establish tight bounds for each of the above described domination variants on a special class of triangulation graphs - the maximal outerplane graphs. These results are summarized on Table 1. Note that, when we are referring to connected, independent, weakly connected and semitotal dominations, $h(n)$ is replaced by $\gamma_{c}(n), i(n), \gamma_{w}(n)$ and $\gamma_{t 2}(n)$, respectively.

As we can see in Fig 1 these parameters are all distinct. This figure shows a maximal outerplanar graph for which red vertices form a semitotal dominating set, $\gamma_{t 2}(G)=10$, and red and green vertices a weakly connected dominating set, $\gamma_{w}(G)=12$. This example can be easily extended to prove that there are graphs with $n=9 k$, such that $\gamma_{t 2}(G)=2 k$ and $\gamma_{w}(G)=2 k+\left\lfloor\frac{k}{2}\right\rfloor$. On the other hand, orange vertices form a connected dominating set, then $\gamma_{c}(G)=4 k$ 


\begin{tabular}{|c|c|c|c|}
\hline Domination & Connected & $\begin{array}{c}\text { Weakly Connected, } \\
\text { Semitotal or } \\
\text { Independent }\end{array}$ & Total \\
\hline $\begin{array}{c}\gamma(n)=\left\lfloor\frac{n}{3}\right\rfloor[9] \\
\gamma(n)=\left\lfloor\frac{n+n_{2}}{4}\right\rfloor^{\mathrm{a}}[11]\end{array}$ & $\gamma_{c}(n)=\left\lfloor\frac{n-2}{2}\right\rfloor$ & $\begin{array}{c}\gamma_{w}(n)=\gamma_{t 2}(n)=i(n)=\left\lfloor\frac{n}{3}\right\rfloor \\
\gamma_{w}(n)=\gamma_{t 2}(n)=i(n)=\left\lfloor\frac{n+n_{2}}{4}\right\rfloor\end{array}$ & $\gamma_{t}(n)=\left\lfloor\frac{2 n}{5}\right\rfloor[3]$ \\
\hline
\end{tabular}

${ }^{\text {a }} n_{2}$ is the number of vertices of degree 2 .

Table 1

A summary of the results related to domination and its variants for maximal outerplanar graphs.

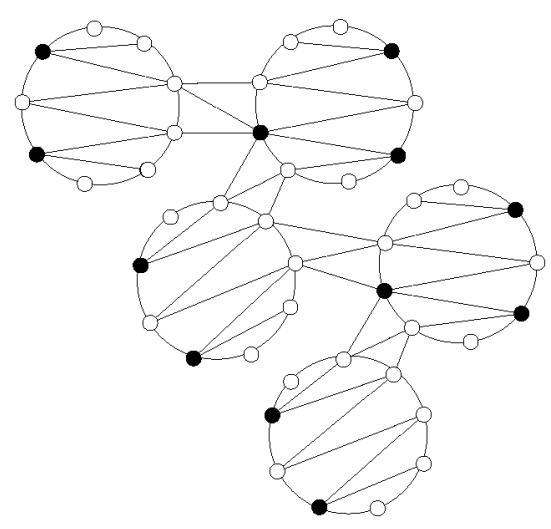

(a)

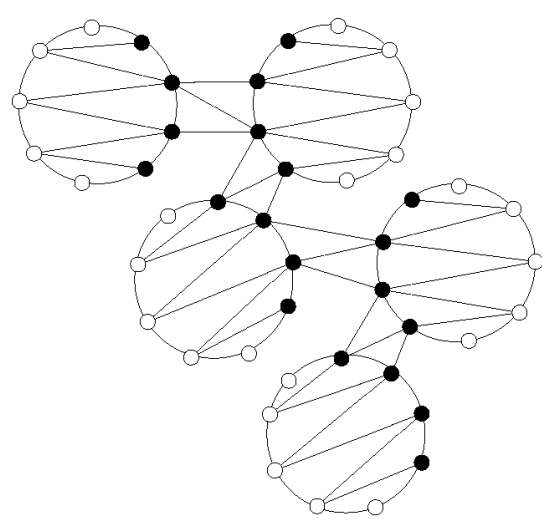

(b)

Fig. 1. A maximal outerplanar graph such that: (a) $\gamma_{t 2}(G)=10$ and $\gamma_{w}(G)=12$; (b) $\gamma_{c}(G)=20$.

\section{Connected Domination and Independent Domination}

We start by relating the concepts of connected domination and connected guarding. In maximal outerplanar graphs these two problems are equivalent. Being $G=(V, E)$, a subset $S \subset V$ is a connected guarding set if every triangle of $G$ has a vertex in $S$ and $G[S]$ is a connected graph. The minimum number of elements in a connected guarding set is denoted by $g_{c}(G)$.

Lemma 3.1 (Pinciu [10]) Let $G=(V, E)$ be a maximal outerplanar graph and $S \subset V . S$ is connected dominant if and only if $S$ is a connected guarding set.

In 1993, Hernández [8] proved that $g_{c}(n)=\left\lfloor\frac{n-2}{2}\right\rfloor$, being $g_{c}(n)=\max \left\{g_{c}(G)\right.$ : $G$ is a maximal outerplanar graph with $n$ vertices $\}$, thus $\gamma_{c}(n)=\left\lfloor\frac{n-2}{2}\right\rfloor$. Now, we are going to study the combinatorial bounds for $i(n)$ in maximal outerplanar graphs. Let $G$ be a maximal outerplanar graph, $G$ is 3-colorable and the 
vertices of any color is a dominating independent set. Selecting the vertices of the least frequently used color we have a $i(G) \leq\left\lfloor\frac{n}{3}\right\rfloor$. Also this bound is tight, i. e., $i(n)=\left\lfloor\frac{n}{3}\right\rfloor$. And can also be expressed as a function of the vertices of degree two, following Tokunaga [11], $i(n)=\left\lfloor\frac{n+n_{2}}{4}\right\rfloor$.

\section{Weakly Connected Domination and Semitotal Dom- ination}

In this section we determine combinatorial bounds for weakly connected and semitotal domination variants on triangulation graphs, because the bounds (and proofs) are the same as for maximal outerplanar graphs. And as the results are the same for both types of domination so we present them together.

Theorem 4.1 If $G$ is a triangulation graph with $n>5$ vertices, then $\gamma_{t 2}(G) \leq$ $\gamma_{w}(G) \leq\left\lfloor\frac{n}{3}\right\rfloor$.

Proof (Sketch) The first inequality is true by definition. To prove the second one, we follow the ideas of Matheson [9], proving that any $n$-vertex triangulation graph $G$ admits a 3-coloring (not necessarily a proper coloring) that verifies: (1) the 3-coloring of the outer face vertices is proper; and (2) the vertices of any color form a weakly (semitotal) dominating set of $G$.

The above bounds are tight as shown by the graphs in Figure 2, then $\gamma_{t 2}(n)=\gamma_{w}(n)=\left\lfloor\frac{n}{3}\right\rfloor$.

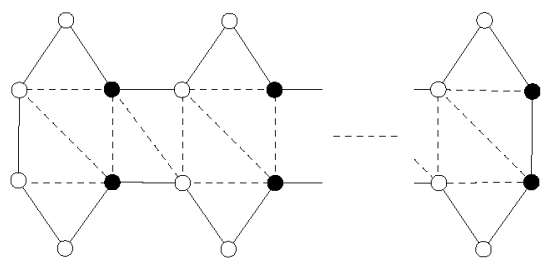

Fig. 2. A maximal outerplanar graph $G$ such that $\gamma_{t 2}(G) \geq\left\lfloor\frac{n}{3}\right\rfloor$.

Theorem 4.2 If $G$ is a maximal outerplanar graph of order $n>5$, then $\gamma_{t 2}(G) \leq \gamma_{w}(G) \leq\left\lfloor\frac{n+n_{2}}{4}\right\rfloor$.

The proof (omitted) is based on the following lemma:

Lemma 4.3 (Tokunaga [11]) Any maximal outerplanar graph $G$ can be 4-colored such that every cycle of length 4 in $G$ has all four colors. 


\section{$5 \quad$ Algorithmic Results}

The domination problems in graphs are, in general, NP-complete. Some variants of domination also remain NP-complete problems, see $[4,7]$. However, there are linear algorithms that solve the domination problem in graphs with bounded treewidth, and the outerplanar graphs have treewidth equal to 2 . These algorithms can be extended without difficulty to solve the variants studied in this work. In addition, we have studied very simple algorithms for special types of outerplanar graphs.

\section{References}

[1] Canales S., Hernández G., Martins M., Matos I., Distance domination, guarding and vertex cover for maximal outerplanar graphs, Discrete Applied Mathematics, 181 (2015), pp. 41-49.

[2] Campos C.N., Wakabayashi Y., On dominating sets of maximal outerplanar graphs, Discrete Appl.Math, 161(3) (2013), 330-335.

[3] Dorfling M., Hattingh, J. H., Jonck E., Total domination in maximal outerplanar graphs II. Discrete Math. 339(3) (2016), 1180-1188.

[4] Garey, M. R. and Johnson, D.S., "Computers and Intractability: A Guide to the Theory of NP-Completeness", W. H. Freeman, 1979.

[5] Goddard, W., Henning, M.A., McPillan, C.A., Semitotal domination in graphs, Util. Math., 94 (2014), 67-81.

[6] Grossman, J.W., Dominating sets whose closed stars form spanning trees, Discrete Math., 169 (1997) 83-94.

[7] Haynes, T.W., Hedetniemi, S., Slater, P., "Fundamentals of Domination in Graphs," Marcel Dekker, New York, 1998.

[8] Hernández G., Controlling guards, Proc. of the Sixth Canadian Conference on Computational Geometry, Saskatoon (Canada) (1994), 387-392

[9] Matheson L. R., Tarjan R. E., Dominating Sets in Planar Graphs, European Journal of Combinatorics, 17(6) (1996), 565-568.

[10] Pinciu, V., "Dominating sets for outerplanar graphs", WSEAS Transactions on Mathematics, 3 (2004), 55-58

[11] Tokunaga, S., Dominating sets of maximal outerplanar graphs, Discrete Appl.Math, 161(18) (2013), 3097-3099. 